\title{
High-Resolution CT Findings in Children with a Normal Pinna or Grade I Microtia and Unilateral Mild Stenosis of the External Auditory Canal
}

\author{
R. Jacob, S. Gupta, B. Isaacson, J.W. Kutz Jr, P. Roland, Y. Xi, and T.N. Booth
}

\begin{abstract}
SUMMARY: A subset of patients presents with unilateral conductive hearing loss, a normal pinna or grade I microtia, and mild external auditory canal stenosis. The physical findings of microtia and a small external canal are commonly absent or subtle in this group of patients, who are being commonly referred for imaging to evaluate isolated conductive hearing loss. We present a case series of patients with unilateral conductive hearing loss and characteristic ossicular abnormalities, commonly anterior fixation of the malleus. All patients had a significantly increased distance from the cochlear promontory to the handle of the malleus and an abnormal incudostapedial angle, indicative of an abnormal ossicular position and/or morphology. Successful surgical reconstruction of the ossicular chain was attempted and accomplished in 3 patients.
\end{abstract}

ABBREVIATIONS: $\mathrm{CHL}=$ conductive hearing loss; $\mathrm{EAC}=$ external auditory canal; $\mathrm{HRCT}=$ high-resolution $\mathrm{CT}$

$C^{\circ}$ ongenital hearing loss affects $1-3$ per 1000 children. ${ }^{1}$ Hearing loss can be due to central auditory dysfunction, conductive, sensorineural, or a combination. ${ }^{2}$ Congenital conductive hearing loss (CHL) may be related to an isolated anomaly of the middle ear without associated anomalies of the external ear or canal. ${ }^{3}$ More commonly, anomalies of the middle ear are linked to congenital anomalies of the pinna, which has an incidence of approximately 1 in 11,000 children. These patients present with conductive hearing loss related to stenosis or atresia of the external auditory canal (EAC). ${ }^{4}$ Ossicular anomalies such as absence, fixation, and abnormal fusion have been described in children with or without EAC stenosis or atresia. External auditory canal stenosis or atresia in the absence of anomalies of the pinna is uncommon but has been described in nonsyndromic children and children with chromosome $18 \mathrm{q}$ deletion. ${ }^{5}$ The absence or subtle nature of external ear anomalies in this patient population could potentially lead to delay in identification of the etiology of hearing loss. High-resolution CT (HRCT) is beneficial in elucidating the

Received May 6, 2014; accepted after revision June 22.

From the Departments of Radiology (R.J., Y.X., T.N.B.) and Otolaryngology (S.G., B.I., J.W.K., P.R.), Children's Medical Center of Dallas, University of Texas, Southwestern Medical Center, Dallas, Texas.

Paper previously presented at: Annual Meeting of the American Society of Neuroradiology and the Foundation of the ASNR Symposium, May 17-22, 2014; Montreal, Quebec, Canada.

Please address correspondence to Timothy N. Booth, MD, Department of Radiology, Children's Medical Center of Dallas, 1935 Medical District Dr, Dallas, TX 75235; e-mail: tim.booth@childrens.com

http://dx.doi.org/10.3174/ajnr.A4067 etiology of congenital CHL and guides surgical management in these patients. ${ }^{4}$

We describe the clinical presentation and audiometric and HRCT findings in a group of patients with unilateral CHL and a normal or minimally abnormal pinna associated with mild stenosis of the EAC.

\section{Case Series}

Patients. This was a Health Insurance Portability and Accountability Act-compliant retrospective case series performed after approval from the institutional review board at a tertiary referral center. A PACS search was performed with the Boolean function combining "external canal," "external auditory canal" AND "stenosis," or "stenotic" for identifying relevant cases of CT of the temporal bones performed during a 5-year period (2009-2013). The inclusion criteria were patients with unilateral congenital EAC stenosis of $<50 \%$ diameter on CT, a normal pinna or grade I microtia, and a diagnostic temporal bone HRCT. Children with syndromes associated with temporal bone abnormalities were excluded. Grade I microtia is characterized by a hypoplastic auricle with all the identifiable landmarks, grade II microtia consists of an abnormal auricle without some identifiable landmarks, grade III microtia is recognized by a very small auricular tag, and anotia is classified as grade IV. , $^{\text {7 }}$

The electronic medical records were reviewed for the presence or absence of an abnormal pinna. Audiograms were reviewed for the severity of conductive hearing loss, and the pure tone average was graded as mild $(20-40 \mathrm{~dB})$, moderate $(40-60 \mathrm{~dB})$, or severe/ 

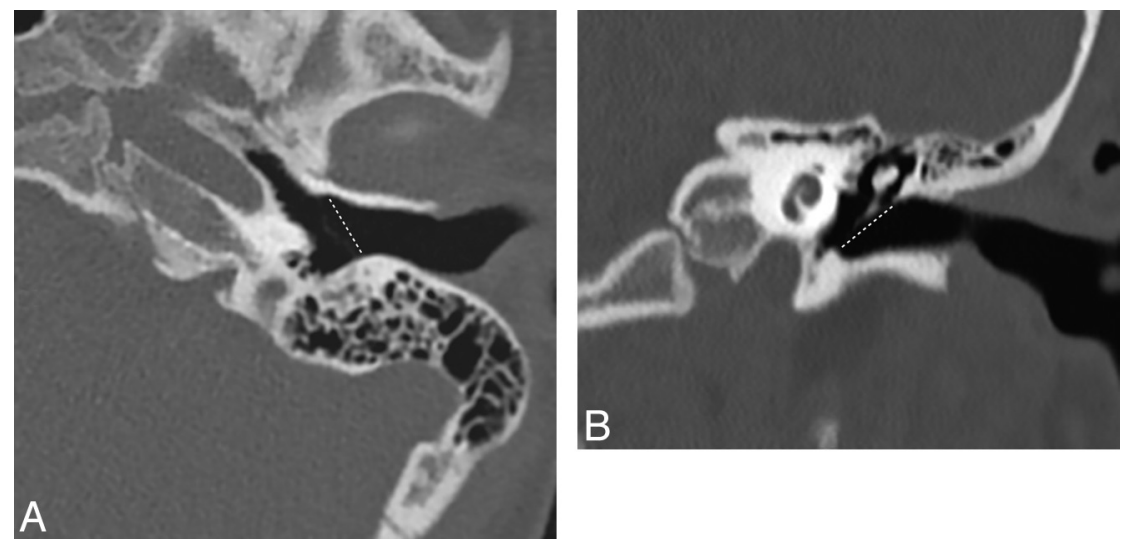

FIG 1. Measurement method for the EAC diameter. Axial and coronal temporal bones at the level of the EAC. The left EAC is measured obliquely along the most medial aspect, parallel to the tympanic membrane (dotted line) in both the axial and coronal planes.
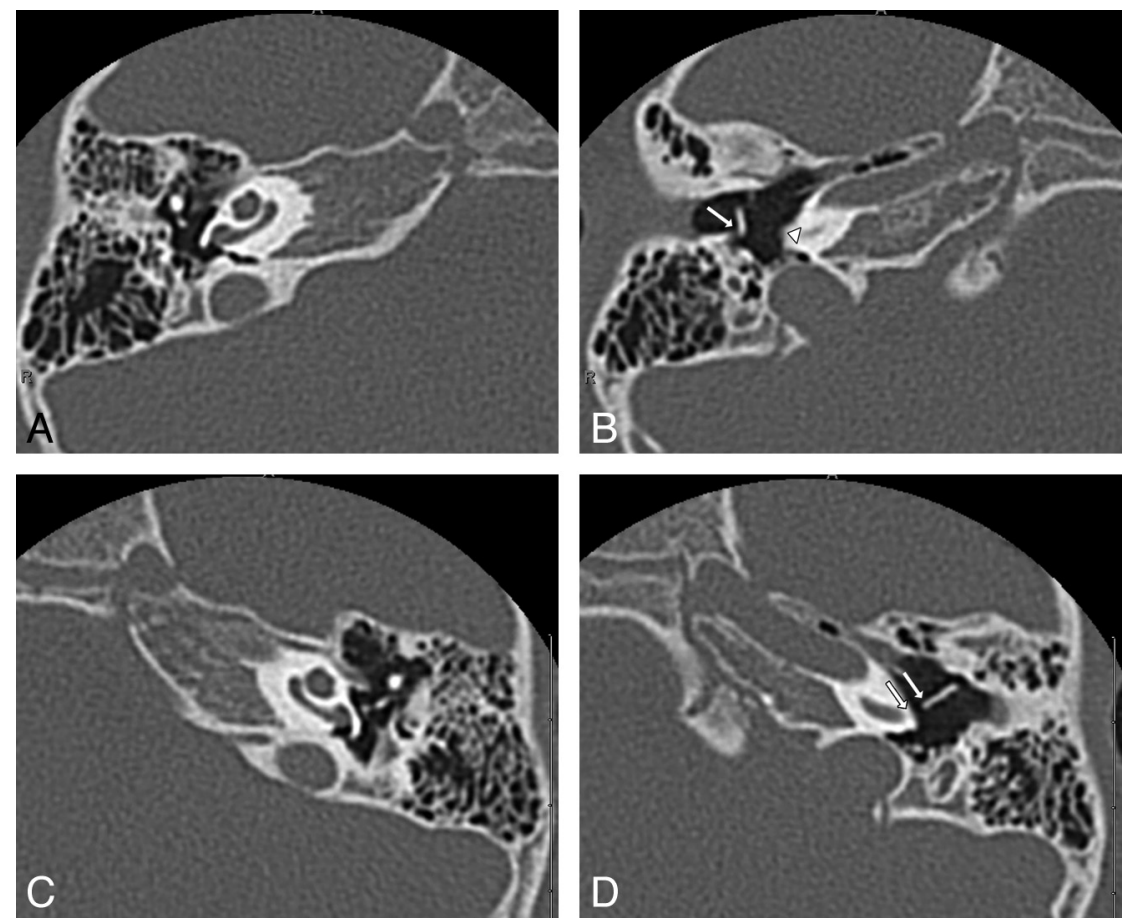

FIG 2. Abnormal orientation of the handle of the malleus in a 4-year-old girl with right-sided $\mathrm{CHL}$ (patient 6). Axial HRCT of the temporal bone, superior-to-inferior. $A$ and $B$, Superior image shows a normal ossicular position. Inferiorly, abnormal orientation of the handle of the right malleus along with posterior fixation of the handle of the malleus (arrow) is present. There is asymmetric widening of the distance between the cochlear promontory (arrowhead) and the handle of the malleus. $C$ and $D$, Normal ossicular anatomy is present superiorly. Inferiorly, normal orientation of the handle of the left malleus and a normal distance from the promontory to the handle (between arrows) are seen.

profound ( $\geq 60 \mathrm{~dB}$ ). HRCT studies were reviewed by a pediatric neuroradiologist with 17 years of experience and a pediatric neuroradiology fellow.

\section{Imaging Analysis}

HRCT studies were performed with a reconstruction thickness of 0.5-0.8 mm. Targeted magnified reconstructions were performed for each ear in the plane of the lateral semicircular canal, with perpendicular coronal reformats constructed at $0.2-$ to 0.5 -mm thickness.

The external auditory canal measurement was compared with that of the contralateral normal ear, which served as an internal standard. The EAC was measured in the axial and coronal planes, parallel to the tympanic membrane, along the medial-most aspect of the external auditory canal (Fig 1). The measurements were compared with the contralateral EAC to assess the degree of asymmetry in size. More complex methods have been described, but a single axial and coronal measurement was determined adequate due to the availability of the contralateral normal ear for comparison. ${ }^{8}$

HRCT of the temporal bone was evaluated for findings based on the criteria developed by Jahrsdoerfer et $\mathrm{al}^{9}$ for identifying suitable candidates with congenital aural atresia for surgery. ${ }^{10,11}$ The presence of ossicular anomalies, including fixation, abnormal position, and abnormal shape or size, was noted. Fixation was defined as the presence of an osseous bar between the ossicles and the margins of the middle ear cavity or when the ossicles were not separable from the walls of the middle ear cavity. The positions and relationships of the ossicles in the middle ear were evaluated. The distance between the cochlear promontory and the most medial portion of malleus handle was measured on the normal and abnormal sides on axial HRCT images to assess the position of the malleus. To evaluate the relationship between the incus and stapes, we measured the angle between the long process of the incus and the capitulum stapes (incudostapedial angle) on the normal and abnormal sides on coronal images at the level of the oval window. ${ }^{10,12}$ The size of the Prussak space was compared with that of the contralateral normal ear. The incudomalleal and incudostapedial joints were evaluated for fusion or widening. The size of the middle ear was compared with that of the contralateral ear and subjectively evaluated as normal or mildly or severely hypoplastic.

The patency of the oval and round windows was assessed along with the status of the facial nerve. The position of the tympanic segment of the facial nerve was considered abnormal if it was inferiorly medially displaced, overlying the oval window; if the position of the posterior genu was anterior to the round window; and/or if the vertical segment of the facial canal had an anterolateral course. Inner ear structures were evaluated for the presence of cochlear or vestibular dysplasia and the size of the cochlear nerve canal (normal, $>1.4 \mathrm{~mm}$ ). ${ }^{13}$ Intraoperative findings, audiograms, and the success of reconstruction were reviewed by a neurotologist. 

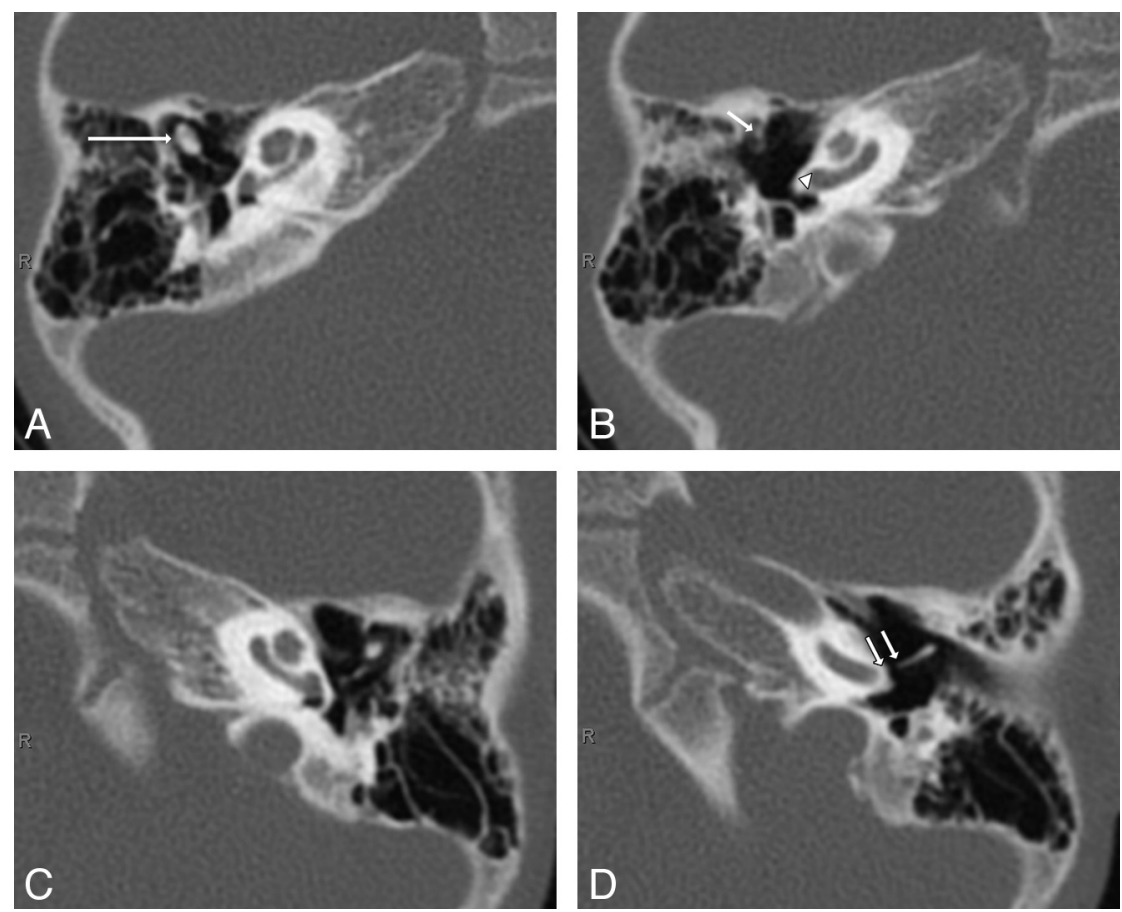

FIG 3. Abnormal fixation of the handle of the malleus along with increased distance of the handle of malleus to the cochlear promontory in a 4-year-old boy with right-sided CHL (patient 9). Axial HRCT of the temporal bones, superior-to-inferior. $A$ and $B$, There is abnormal fusion of the right malleus and incus (long arrow) superiorly. The inferior image shows fixation of the handle of the malleus anteriorly (short arrow). Note an increased distance of the handle of the malleus to the cochlear promontory (arrowhead). $C$ and D. Normal separation of the left ossicles is present superiorly. Inferiorly, there is a normal distance of the handle of the left malleus to the promontory in the normal ear (between arrows).
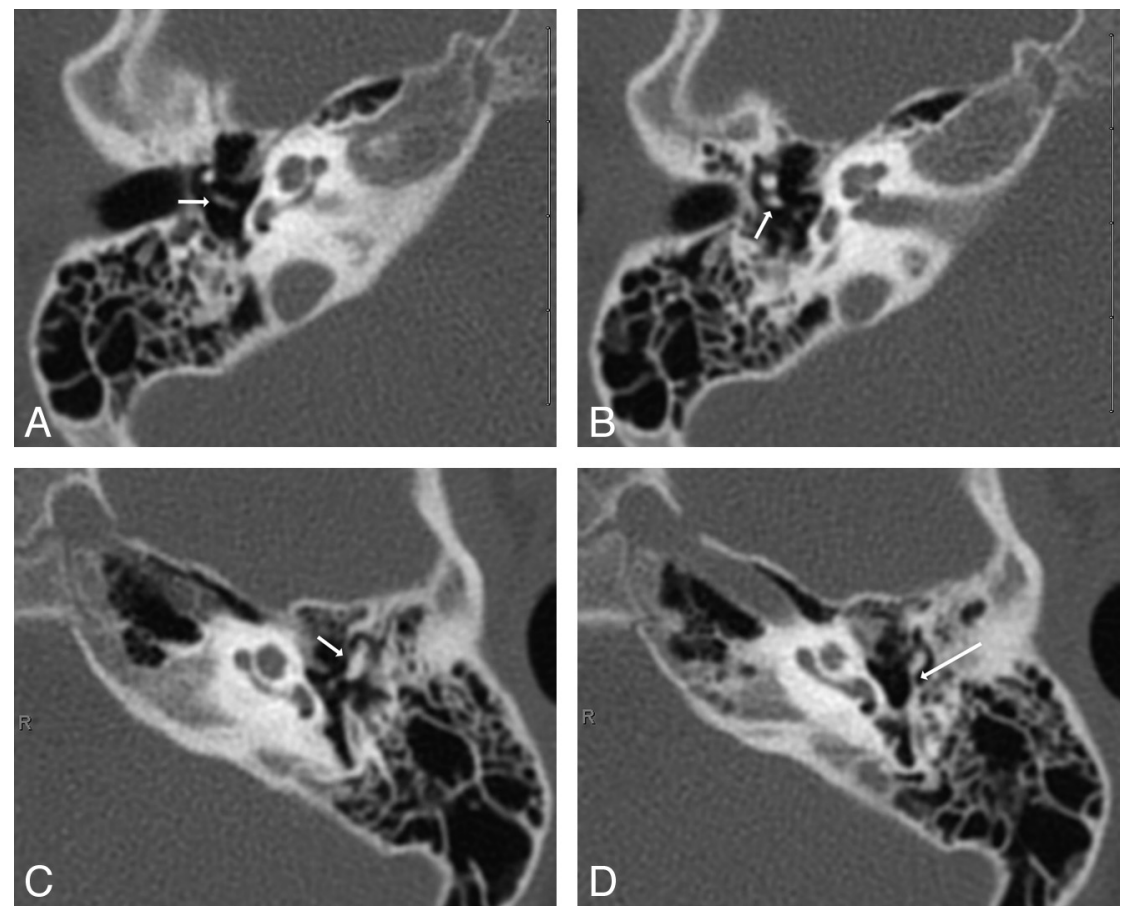

FIG 4. Abnormal fixation of the incus in a 5-year-old girl with left-sided $\mathrm{CHL}$ (patient 1). Axial HRCT of the temporal bone, superior-to-inferior. $A$ and $B$, Superiorly, there is a normal incus with articulation with the stapes (arrow). Inferior image shows a normal long process of the incus with no fixation bar (arrow). $C$ and D, Superiorly, there is fusion of the left malleus and incus (arrow). Inferior image shows a posterior fixation bar extending from the long process of the incus (long arrow) to the posterior wall of the tympanic cavity.
Statistical analysis was performed by using the Wilcoxon signed rank test due to the small sample size. The malleal handle to the cochlear promontory distance and the incudostapedial angle were compared with the measurements from the contralateral normal ear.

\section{Clinical Findings}

A 5-year PACS search with specified criteria revealed 9 patients. Three were excluded due to syndromic association (2 Goldenhar, 1 Down syndrome). The male/female ratio was 5:4, and the mean age was 7 years (range, 3-12 years). All patients presented with unilateral CHL. On physical examination, 5/9 patients had no abnormality of the pinna and clinically normal EACs. Four of 9 patients had grade I microtia. The mean pure tone average was $49.4 \pm 14.2 \mathrm{~dB}$ (range, 28-68 $\mathrm{dB})$, and the mean air-bone gap was $49.6 \pm 12.9 \mathrm{~dB}$ (range, 25-65 dB). Two patients had mild CHL; 5 , moderate CHL; and 2, severe CHL.

\section{Imaging Findings}

CT showed the mean EAC diameter to be $5.1 / 6.7 \mathrm{~mm}$ (axial/coronal) on the affected side and 7.7/8.7 $\mathrm{mm}$ (axial/coronal) in the normal-hearing ear. Fixation of the handle of the malleus to the tympanic annulus was seen anteriorly in 7/9 ears and posteriorly in 1 ear (Figs 2 and 3 ). Posterior fixation of the long process of the incus was present in 2/9 ears (Fig 4). Abnormal orientation of the handle of the malleus with an abnormally increased distance to the cochlear promontory was present in all ears. The mean distance between the cochlear promontory and the handle of the malleus on the normal side was $1.7 \mathrm{~mm}$ (range, 1-2.9 mm) and 3.9 $\mathrm{mm}$ (range, 3.1-4.4 $\mathrm{mm}$ ) on the abnormal side, with the distance being increased on the abnormal side (Figs 2 and $3)$. These findings were found to be significant $(P=.039$, Wilcoxon signed rank test). The mean incudostapedial angle on the normal side was $82.3^{\circ}$ (range, $66^{\circ}-$ $96^{\circ}$ ) and $101.1^{\circ}$ (range, $73^{\circ}-119^{\circ}$ ) on the abnormal side (Fig 5). These findings were significant $(P=.015$, Wilcoxon signed rank test). The angle was increased in all affected ears.

There was a mild subjective decrease in size in the middle ear on the affected 

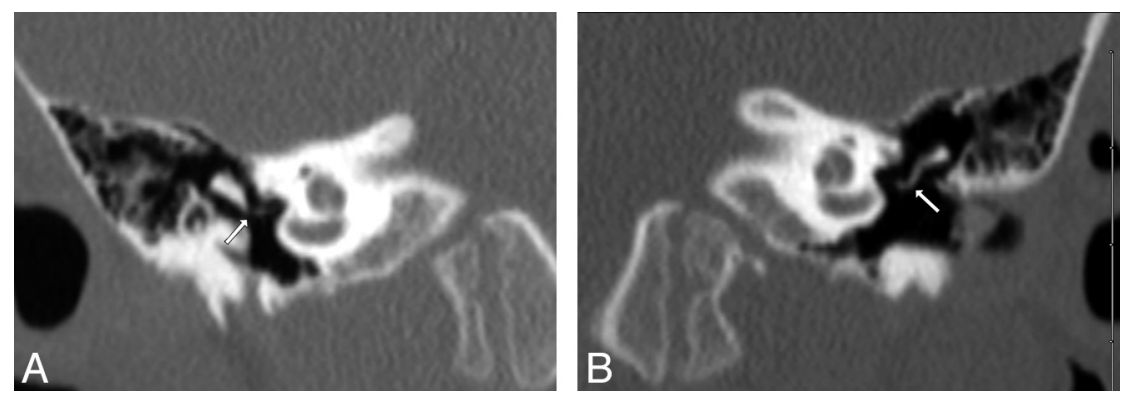

FIG 5. Abnormal asymmetric increased in the incudostapedial angle in a 3-year-old boy with right-sided CHL (patient 8). Coronal-reconstruction HRCT of the temporal bone at the level of the long process of the incus. $A$, An abnormally increased incudostapedial angle (arrow) measuring $109^{\circ}$ is present in the right ear. $B$, The normal left ear has an incudostapedial angle measuring $93^{\circ}$ (arrow).
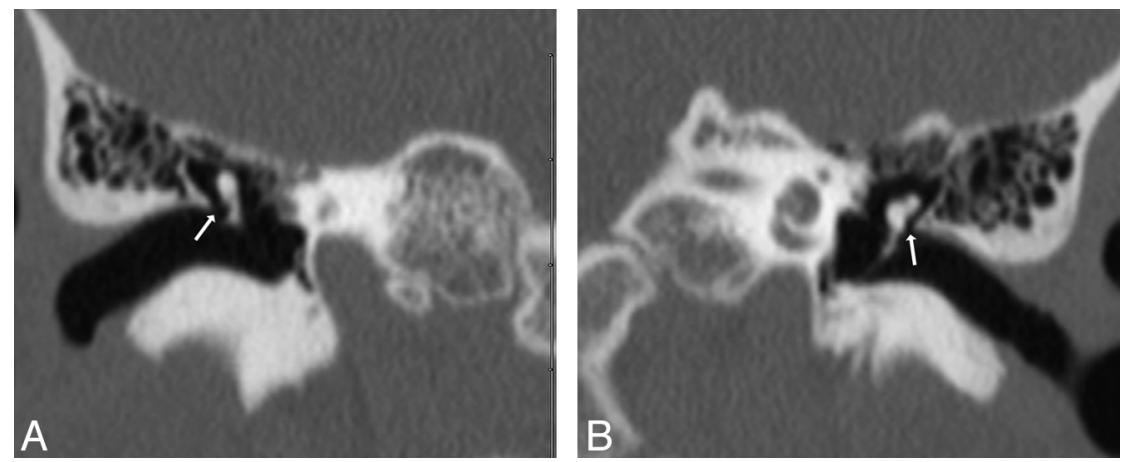

FIG 6. Asymmetric narrowing of the Prussak space in a 12-year-old boy with left-sided CHL (patient 2). Coronal-reconstruction HRCT of the temporal bone at the level of the malleus. $A$, Normal appearance of the Prussak space in the normal right ear (arrow) is present. B, Asymmetric narrowing of the Prussak space (arrow) in the affected left ear is present with a normal volume of the tympanic cavity.
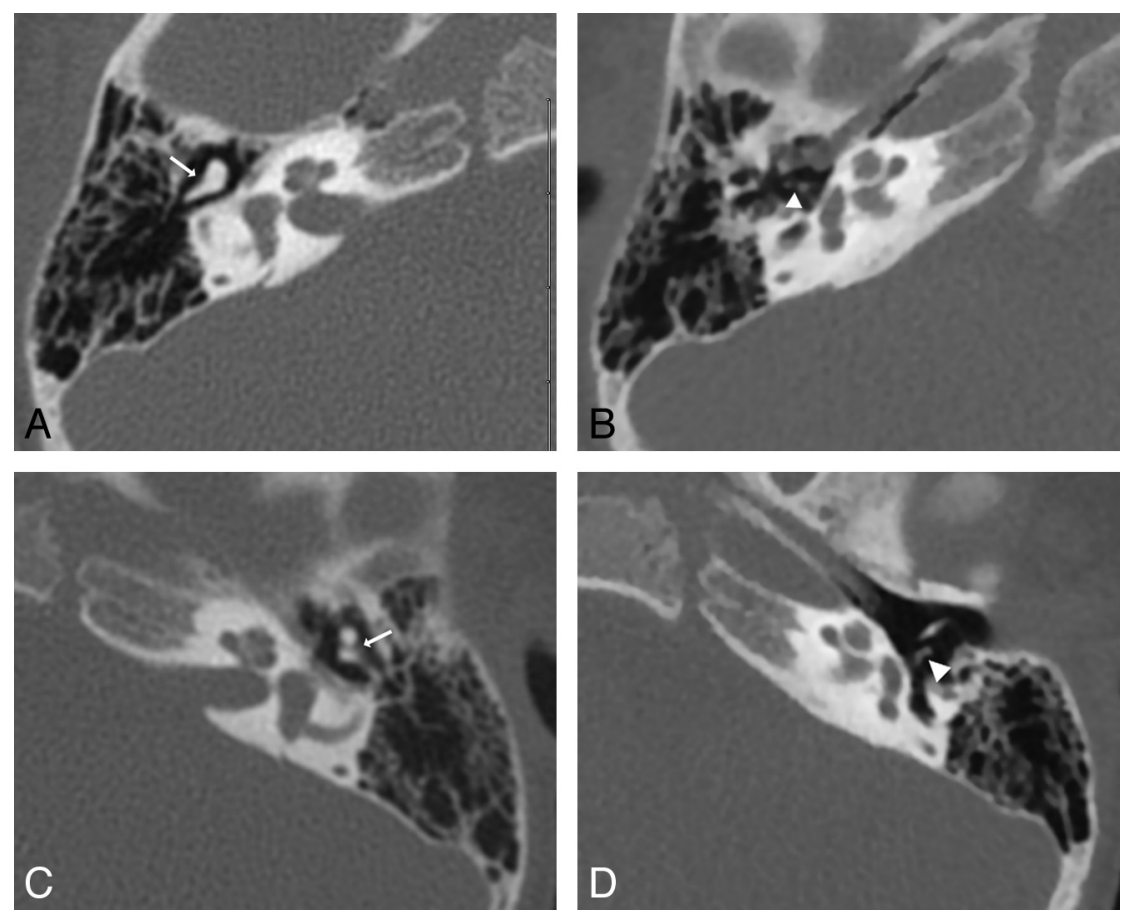

FIG 7. Abnormal incudomalleal and incudostapedial joints in a 3-year-old boy with right-sided CHL (patient 8). Axial HRCT of the temporal bone at the level of the ossicular articulations, superior-toinferior. $A$ and $B$, Superior image shows abnormal incudomalleal fusion (arrow). Inferiorly, abnormal incudostapedial widening (arrowhead) in the right ear is present. $C$ and $D$, The normal left incudomalleal (arrow) and incudostapedial joints (arrowhead) are demonstrated. side in 5 ears, and the middle ear was nor$\mathrm{mal}$ in size in 4 ears. There were no patients with severe hypoplasia of the middle ear cavity, and all ears were normally aerated without soft tissue present. A narrowed Prussak space suggested an abnormal ossicular position in 5/9 ears (Fig 6). The most common ossicular abnormalities included decreased size of the malleus head (7/9) and a narrowed or fused incudomalleal joint (7/9). Widening of the incudostapedial joint was present in 5/9 ears (Fig 7). The stapes was abnormal in 1 patient with an enlarged capitulum.

The oval and round windows were normal in all ears. The tympanic segment of the facial nerve was normally positioned and free of the oval window in all ears. The posterior genu was in the normal position at the level of or posterior to the round window in all ears. There was mild anterior displacement of the vertical segment of the facial nerve in 1 ear. No inner ear anomalies and normal cochlear nerve canals were demonstrated.

\section{Surgical Correlation}

Surgery was performed in 3 patients with agreement of most findings on HRCT. One imaging study showed a widened incudostapedial joint, which was not apparent at surgery. Tympanoplasty was performed in all those with a canaloplasty in 1 ear. Natural ossiculoplasty (mobilization of the fixed malleus head) was performed in 2 ears with malleus fixation, and an ossicular chain reconstruction (partial ossicular reconstruction prosthesis) was performed in 1 ear with incudostapedial joint separation. Significant improvement in hearing was noted in 2 patients on the postoperative audiogram (mean improvements in pure tone average of $24 \mathrm{~dB}$ ), and 1 patient was lost to follow-up.

\section{DISCUSSION}

The auricle is derived from the first and second pharyngeal arches, and the external auditory canal is derived from the first pharyngeal cleft. The malleus and incus are derived from endochondral ossification of the cartilage of the first and second arches. ${ }^{4}$ Hence, abnormal development of the first and second arches can lead to auricular malformations, including microtia, external auditory canal stenosis, 
and ossicular dysplasia. The early identification of unilateral conductive hearing loss can be difficult in the absence or presence of minor deformity of the external ear. ${ }^{5}$

Most patients in this series had normal external examination findings on the initial evaluation when unilateral CHL was the sole reason for obtaining HRCT. 3D volumetric reconstruction of the CT dataset may be useful in assessing minor external ear malformations to aid in a potential diagnosis. Bilateral EAC stenosis would be more difficult to evaluate by imaging without the contralateral ear for comparison. However, most patients, approximately two-thirds, with congenital EAC stenosis have unilateral disease, which would facilitate an imaging diagnosis. ${ }^{14}$ Patients with syndromic associations are also more commonly associated with bilateral involvement and would be identified clinically. ${ }^{15}$ Patients presenting with unilateral CHL should be evaluated for mild stenosis of the external auditory canal on HRCT, especially because anomalies of the external ear can be subtle or absent on physical examination. Development of a simple normative EAC diameter may be helpful to evaluate the child with bilateral CHL.

Middle ear and ossicular anomalies are more common in children presenting with CHL and EAC stenosis. ${ }^{15}$ The present study identified characteristic fixation abnormalities of the ossicles in this group of patients. High-resolution CT in the present study demonstrated that the malleus handle was laterally displaced from the cochlear promontory. Most commonly, this displacement appears to relate to anterior rotation of the handle with anterior fixation to the anterior tympanic annulus. Additional indirect signs such as narrowing of the Prussak space on the affected side can be very helpful in identifying ossicular anomalies if they are not readily apparent. The incudostapedial angle was also consistently increased on the affected side but remained $<120^{\circ}$ in all affected ears. An incudostapedial angle of $<120^{\circ}$ has been associated with a good surgical prognosis. ${ }^{16}$

Mayer et $\mathrm{al}^{10}$ found the oval window to be absent in one-third of the patients with EAC stenosis or atresia and noted no significant correlation with the degree of associated external ear abnormality. A main predictor of the success of ossicular reconstruction is the patency of the oval window, and all were widely patent in the present study. ${ }^{4}$ The limited number in our group that had surgery had excellent hearing results.

Malposition of the facial nerve has been reported in mild microtia in up to half of patients. ${ }^{9}$ This is typically manifested by anterior displacement of the mastoid segment. Only 1 patient in this series had a mild anteriorly positioned mastoid segment. The obstruction of access to the oval window by the anterolateral displacement of the vertical segment of the facial canal was reported in $41 \%$ of patients with atresia in the series by Dedhia et al. ${ }^{11}$ The abnormal position of the facial nerve could potentially increase the risk of injury to the nerve. There were no anomalies of the tympanic segment of the facial nerve canal in this series, which would have precluded surgical access to the oval window.

Potential limitations of our study include the use of search criteria based on radiologic reports. This may significantly underestimate the incidence of such findings because the presence of mild stenosis may be difficult to subjectively appreciate. The study is also limited by the resultant small sample size and surgical correlation in only 3 patients.

\section{CONCLUSIONS}

Patients presenting with unilateral conductive hearing loss should be evaluated for mild EAC stenosis and potentially subtle but characteristic ossicular malformations. Anomalies of the pinna are commonly absent or subtle in this group of patients. Systematic evaluation of the middle ear structures is suggested with evaluation of the distance from the cochlear promontory to the handle of the malleus and the incudostapedial angle. These patients typically have favorable anatomy for ossicular reconstruction.

Disclosures: Brandon Isaacson—UNRELATED: Board Membership: Advanced Bionics, Comments: Medical Advisory Board; Consultancy, Medtronic Midas Rex Institute, Comments: consultant for drill design; Payment for Lectures (including service on Speakers Bureaus): Oticon, Comments: lectured on bone-anchored hearing aid; Travel/Accommodations/Meeting Expenses Unrelated to Activities Listed: Oticon, Comments: discussed bone-anchored hearing aids and cochlear implants in Copenhagen, Denmark and Nice, France.

\section{REFERENCES}

1. Kemper AR, Downs SM. A cost-effectiveness analysis of newborn hearing screening strategies. Arch Pediatr Adolesc Med 2000;154: 484-88

2. Smith RJ, Shearer AE, Hildebrand MS, et al. Deafness and hereditary hearing loss overview. February 14, 1999; updated January 9, 2014. In: Pagon RA, Adam MP, Bird TD, et al, eds. GeneReviews. Seattle: University of Washington, Seattle; 1993-2014. http://www.ncbi.nlm. nih.gov/books/NBK1434/. Accessed April 21, 2014

3. Park K, Choung YH. Isolated congenital ossicular anomalies. Acta Otolaryngol 2009;129:419-22

4. Raz Y, Lustig L. Surgical management of conductive hearing loss in children. Otolaryngol Clin North Am 2002;35:853-75

5. Stewart JM, Downs MP. Congenital conductive hearing loss: the need for early identification and intervention. Pediatrics 1993;91: 355-59

6. Marx H. Die Missbildungen des Ohres. In: Henke F, Lubarsh O, eds. Handbuch der Spez Path Anatomie Histologie. Berlin: Springer-Verlag; 1926:620-25

7. Ishimoto S, Ito K, Yamasoba T, et al. Correlation between microtia and temporal bone malformation evaluated using grading systems. Arch Otolaryngol Head Neck Surg 2005;131:326-29

8. Mahboubi H, Wu EC, Jahanbakhshi R, et al. A novel method to determine standardized anatomic dimensions of the osseous external auditory canal. Otol Neurotol 2012;33:715-20

9. Jahrsdoerfer RA, Yeakley JW, Aguilar EA, et al. Grading system for the selection of patients with congenital aural atresia. Am J Otol 1992;13:6-12

10. Mayer TE, Brueckmann H, Siegert R, et al. High-resolution CT of the temporal bone in dysplasia of the auricle and external auditory canal. AJNR Am J Neuroradiol 1997;18:53-65

11. Dedhia K, Yellon RF, Branstetter BF, et al. Anatomic variants on computed tomography in congenital aural atresia. Otolaryngol Head Neck Surg 2012;147:323-28

12. Skinner M, Honrado C, Prasad M, et al. The incudostapedial joint angle: implications for stapes surgery prosthesis selection and crimping. Laryngoscope 2003;113:647-53

13. Yi JS, Lim HW, Kang BC, et al. Proportion of bony cochlear nerve canal anomalies in unilateral sensorineural hearing loss in children. Int J Pediatr Otorhinolaryngol 2013;77:530-33

14. Gassner EM, Mallouhi A, Jaschke WR. Preoperative evaluation of external auditory canal atresia on high-resolution CT. AJR Am J Roengtenol 2004;182:1305-12

15. Tekes A, Ishman SL, Baugher KM, et al. Does microtia predict severity of temporal bone CT abnormalities in children with persistent conductive hearing loss? J Neuroradiol 2013;40:192-97

16. Kim DW, Lee JH, Song JJ, et al. Continuity of the incudostapedial joint: a novel prognostic factor in postoperative hearing outcomes in congenital aural atresia. Acta Otolaryngol 2011;131:701-07 